\title{
Effect of soluble ST2 as a tool for the evaluation of volume status in kidney transplant recipients
}

\author{
Woo Yeong Park, Hyun Suk Noh, Jungheon Kwon, Jimin Lim, Yaerim Kim, Jin Hyuk Paek, Kyubok Jin, Seungyeup Han
}

Department of Internal Medicine-Nephrology, Keimyung University Dongsan Medical Center, Daegu, Korea

Background: Cardiovascular disease is the leading cause of death in kidney transplant recipients (KTRs) and it is important to evaluate volume status for heart condition. Echocardiography is an important tool for assessing heart condition, but it is expensive and not readily available. In the recent, soluble ST2 (sST2), which is associated with cardiac matrix remodeling by cardiovascular events, has been considered as a novel cardiac biomarker in the general population, but it is uncertain in KTRs. Therefore, we investigated the effect of sST2 as a tool for the evaluation of volume status in KTRs.

Methods: We retrospectively analyzed the medical records of 100 KTRs measured SST2 at Keimyung University Dongsan Hospital between 2019 and 2021. We divided them into higher and lower sST2 groups according to the median value of sST2 of 23.0 $\mathrm{ng} / \mathrm{mL}$. We performed body composition monitor (BCM), echocardiography and cardiac markers such as CK-MB, troponin-I, and NT-proBNP. Hyperhydrated status was defined as the hydration status-to-extracellular water ratio $(\triangle \mathrm{HS})>15 \%$ in $B C M$.

Results: The rate of male sex, dialysis vintage and serum CK-MB level were significantly higher in the higher sST2 group than in the lower sST2 group ( $80 \%$ vs. $42 \%, P<0.001 ; 66.6 \pm 71.3$ vs. $35.0 \pm 47.6$ months, $P=0.014 ; 3.5 \pm 3.0$ vs. $1.5 \pm 0.9 \mathrm{mg} / \mathrm{dL}, \mathrm{P}=0.002)$. Plasma NT-proBNP level, serum troponin-I level, and the rate of hyperhydrated status were higher in the higher sST2 group than in the lower SST2 group, but there was no significant difference between the two groups. Hyperhydrated status, NT-proBNP, CK$M B$, and troponin-I were significantly associated with sST2 levels $(r=0.548, P=0.005 ; r=0.248, P=0.027 ; r=0.415, P=0.006 ; r=0.405$, $\mathrm{P}=0.007$ ). However, the parameters in echocardiography (left ventricular mass index, ejection fraction, and left ventricular and atrial diameters) were not associated with sST2 levels.

Conclusions: SST2, with cardiac markers and BCM, might be effective to evaluate the volume status in KTRs.

Corresponding author: Seungyeup Han

E-mail: hansy@dsmc.or.kr

(c) The Korean Society for Transplantation

This is an Open Access article distributed under the terms of the Creative Commons Attribution Non-Commercial License (http://creativecommons.org/licenses/by-nc/4.0/) which permits unrestricted non-commercial use, distribution, and reproduction in any medium, provided the original work is properly cited. 